\title{
XLII. On the method employed in the trigonometrical survey for finding the length of a degree perpendicular to the meridian
}

\author{
J. Ivory Esq. M.A. F.R.S.
}

To cite this article: J. Ivory Esq. M.A. F.R.S. (1828) XLII. On the method employed in the trigonometrical survey for finding the length of a degree perpendicular to the meridian , Philosophical Magazine Series 2, 4:22, 241-245, DOI: 10.1080/14786442808674803

To link to this article: http://dx.doi.org/10.1080/14786442808674803

曲 Published online: 10 Jul 2009.

Submit your article to this journal $[\pi$

Џll Article views: 2

Q View related articles $\sqsubset$ 
THE

\title{
PHILOSOPHICAL MAGAZINE
}

\author{
AND \\ ANNALS OF PHILOSOPHY. \\ [NEW SERIES.] \\ OCTOBER 1828.
}

XLII. On the Method employed in the Trigonometrical Survey for finding the Length of a Degree perpendicular to the Meridian. By J. Ivony, Esq. M.A. F.R.S.*

IN the first volume of the Trigonometrical Survey, a method $I$ is given for finding the difference of longitude of two stations lying nearly in the same parallel of latitude, without assuming that the earth has any particular figure, and supposing only that it is nearly a sphere + . An important part of the Survey depends upon this method; and, although its accuracy has heretofore been called in question, yet we see from the late volumes of the Phil. Trans. that it is now deemed sufficiently exact and unexceptionable in its principle. It is however remarkable, that this method has never succeeded in practice, or rather, has in every case led to results evidently wide of the truth. This failure it is usual to ascribe to errors in the observed azimuths; but we may reasonably doubt whether this be the sole cause of deficiency, when we observe that the difference of longitude of the two stations, computed by other modes of solution unquestionable in point of accuracy, is always found to depend on the compression of the spheroid. The method of which we are speaking, was first published in the Phil.Trans. for 1790 and 1795 ; it is therefore time to subject to a strict examination a process which, although it has never been of any utility, is still employed in practice; and as I have thrown out some doubts about the grounds of it in this Journal for May last, I deem it incumbent on me to ascertain fully its real character.

Let $\lambda$ and $m$ denote the latitude and azimuth at the first station; $\lambda^{\prime}$ and $m^{\prime}$ the same things at the second station;

* Communicated by the Author.

† Trigonometrical Survey, vol. i. pp. 154, 293 .

Nero Series. Vol. 4. No. 22. Oct. 1828, 2 I 
$\gamma$ the rectilineal distance of the two stations, or the chord between them; $\omega$ the difference of longitude; $a$ the equatorial semidiameter, and $e$ the excentricity of the elliptical meridians. Further, let $\phi$ stand for the angle of depression of the chord below the horizon of the first station: then $\gamma \sin \phi$ will be the perpendicular drawn from the second station upon the horizon of the first; and the distance of this perpendicular from the plane of the meridian of the first station, to which plane it is parallel, will be equal to $\gamma \cos \varphi \sin m$, because the azimuth $m$ is the angle which the projection of the chord upon the horizon makes with the meridian. Now let $x$ be the distance of the second station from the polar axis of the spheroid : then, $\omega$ being the angle between the two meridians, it is obvious that the distance of the second station from the plane of the meridian of the first, will be equal to $x \sin \omega$; and in consequence of what was before proved, we shall have this equation,

$$
x \sin \omega=\gamma \cos \varphi \sin m \text {. }
$$

Next let $R$ be the radius of a sphere which passes through both the stations, and touches the horizon of the first: then,

$$
\operatorname{Sin} \phi=\frac{\gamma}{2 \mathrm{R}}, \cos \varphi=\sqrt{1-\frac{\gamma^{2}}{4 \mathrm{R}^{2}}} .
$$

If we conceive a plane to bisect the chord $\gamma$ at right angles, this plane will cut off from the normal to the earth's surface at the first station, a part equal to the line $R$. Therefore $R$, like the radii of curvature of the spheroid, will always be little different from $a$, the semidiameter of the equator; and since $\gamma$ is always a small part of $\mathrm{R}$, or of $a$, we may substitute $a$ for $\mathbf{R}$ in the expression of $\cos \phi$, without danger of introducing a sensible error in any case that can occur in practice. We have likewise, from the nature of the elliptical spheroid, $x=$ $\frac{a \cos \lambda^{\prime}}{\sqrt{1-e^{2} \sin ^{2} \lambda^{\prime}}}$; and thus we get,

$$
\frac{\cos \lambda^{\prime} \sin \omega}{\sqrt{1-e^{2} \sin ^{2} \lambda^{\prime}}}=\frac{\gamma}{a} \sqrt{1-\frac{\gamma^{2}}{4 a^{2}}} \times \sin m \text {. }
$$

Now, put $\sin \frac{\beta}{2}=\frac{\gamma}{2 a}$; then,

$$
\frac{\gamma}{a} \sqrt{1-\frac{\gamma^{2}}{4 a^{2}}}=2 \sin \frac{\beta}{2} \cos \frac{\beta}{2}=\sin \beta ;
$$

and the last formula will become,

$$
\frac{\cos \lambda^{\prime} \sin \omega}{\sqrt{1-e^{2} \sin 2 \lambda^{\prime}}}=\sin \beta \sin m \text {. }
$$

The like reasoning applied to the second station will furnish another similar equation. 
Thus we finally obtain,

$$
\begin{aligned}
& \operatorname{Cos} \psi^{\prime}=\frac{\cos \lambda^{\prime}}{\sqrt{1-e^{2} \sin ^{2} \lambda^{\prime}}}, \cos \psi^{\prime} \sin \omega=\sin \beta \sin m, \\
& \operatorname{Cos} \psi=\frac{\cos \lambda}{\sqrt{1-e^{2} \sin ^{2} \lambda}}, \cos \psi \sin \omega=\sin \beta \sin m^{\prime} .
\end{aligned}
$$

Suppose now that a spherical triangle is constructed of which the base is equal to the arc $\beta$, and the two sides to the arcs $90-\psi$ and $90-\psi^{\prime}:$ I say, that the angle of this triangle opposite to the base $\beta$ is not sensibly different from $\omega$, the difference of longitude, when the two latitudes are nearly equal; and is exactly equal to it when the two latitudes are equal. In order to prove this, it is to be observed that $a \cos \psi, a \cos \psi^{\prime}$, are the respective distances of the two stations from the polar axis of the spheroid; and $a \sin \psi \sqrt{1-e^{2}}$, $a \sin \psi^{\prime} \sqrt{1-e^{2}}$, are their distances from the plane of the equator. Wherefore, because $w$ is the angle between the two meridians, we have this expression for the square of the chord, viz.

$$
\begin{gathered}
\frac{\gamma^{2}}{a^{2}}=\left(\cos \psi-\cos \psi^{\prime} \cos \omega\right)^{2}+\cos ^{2} \psi^{\prime} \sin ^{2} \omega+\left(1-e^{2}\right)(\sin \psi \\
\left.-\sin \psi^{\prime}\right)^{2} ;
\end{gathered}
$$

or, which is the same thing,

$\frac{\gamma^{2}}{a^{2}}=2-2\left(\cos \psi \cos \psi^{\prime} \cos \omega+\sin \psi \sin \psi^{\prime}\right)-e^{8}\left(\sin \psi-\sin \psi^{\prime}\right)^{8}$ : but $\frac{\gamma^{2}}{a^{2}}=4 \sin ^{8} \frac{\beta}{2}=2(1-\cos \beta)$; and, hence,

$$
\cos \beta=\cos \psi \cos \psi^{\prime} \cos \omega+\sin \psi \sin \psi^{\prime}+\frac{e^{2}}{2}\left(\sin \psi-\sin \psi^{\prime}\right)^{8} \text {. }
$$
Now, from this equation and the relation that is known to subsist between an angle of a spherical triangle and the three sides, it follows that $\omega$ may be reckoned equal to the angle of the triangle opposite to the base $\beta$, whenever the latitudes are so nearly equal that the term multiplied by $e^{2}$, has no sensible value; and when the latitudes are exactly equal, the equality affirmed is rigorously true. But for the greater precision let us inquire, what variation the term multiplied by $e^{2}$ will produce in the arc $\beta$. For this purpose suppose that $\beta$ becomes $\beta+\delta \beta$, then,

$\operatorname{Cos}(\beta+\delta \beta)=\cos \psi \cos \psi^{\prime} \cos \omega+\sin \psi \sin \psi^{\prime}$,

$$
\delta \beta=\frac{\varepsilon\left(\sin \lambda-\sin \lambda^{\prime}\right)}{\sin \overline{1}^{\prime \prime}} \times \frac{a\left(\sin \lambda-\sin \lambda^{\prime}\right)}{\gamma},
$$

the true latitudes being written for $\psi$ and $\psi^{\prime}$, and $\frac{\gamma}{a}$ for $\sin \beta$, in the expression of the small variation. In the instance of Beachy Head and Dunnose, we shall find $\delta \beta=0 " \cdot 11$, a quan- 
tity far below the errors of observation. In this instance, and in all those where this method has been actually used, or where it can be supposed to apply, we may conclude that the angle of the spherical triangle opposite to the base $\beta$, is equal to $\omega$, the difference of longitude of the two stations. But in every spherical triangle, the sines of the angles are proportional to the sines of the opposite sides; and hence, on account of the equations $(x)$, we learn that $m$ is the angle of the same triangle opposite to the side $90-\psi^{\prime}$, and $m^{\prime}$ the angle opposite to the side $90-\psi$.

Having obtained a knowledge of all the parts of the spherical triangle, if we apply to it one of the analogies of Napier, we shall get,

$$
\operatorname{Tan} \frac{\omega}{2}=\frac{\cos \frac{\psi-\psi^{\prime}}{2}}{\sin \frac{\psi+\psi^{\prime}}{2}} \times \operatorname{cotan} \frac{m+m^{\prime}}{2} .
$$

Now this formula is different from the method in the Trigonometrical Survey in no other respect, except that the arcs $\psi$ and $\psi^{\prime}$, which are what are called the reduced latitudes, come in place of the true latitudes $\lambda$ and $\lambda^{\prime}$. But as the reduced latitudes depend upon the excentricity of the spheroid, it follows that the difference of longitude is no more independent of the figure of the earth in this mode of computation, than in any other. Because the difference of latitude is very small, we may write $\cos \frac{\lambda-\lambda^{\prime}}{2}$, or even the radius of the tables, instead of $\cos \frac{\psi-\psi}{2}$ : and I have found, by reducing properly and putting $\varepsilon=\frac{e^{2}}{2}$,

$$
\operatorname{Sin} \frac{\psi+\psi^{\prime}}{2}=\sin \frac{\lambda+\lambda^{\prime}}{2} \times\left(1-\varepsilon \cos ^{2} \frac{\lambda+\lambda^{\prime}}{2}\right) .
$$

The foregoing expression will now become

$$
\operatorname{Tan} \frac{\omega}{2}=\frac{\cos \frac{\lambda-\lambda^{\prime}}{2}}{\sin \frac{\lambda+\lambda^{\prime}}{2}} \times \operatorname{cotan} \frac{m+m^{\prime}}{2} \times\left(1+\varepsilon \cos ^{2} \frac{\lambda+\lambda^{\prime}}{2}\right) ;
$$

or, in logarithms,

$\log \tan . \frac{\omega}{2}=\log \left(\frac{\cos \frac{\lambda-\lambda^{\prime}}{2}}{\sin \frac{\lambda+\lambda^{\prime}}{2}} \times \operatorname{cotan} \frac{m+m}{2}\right)+M \varepsilon \cos ^{2} \frac{\lambda+\lambda^{\prime}}{2}$.

This, then, is the formula by which we must compute the difference of longitude on a spheroid of which $\mathrm{t}$ is the compression; and if we make $\varepsilon=0$, it will coincide with the method 
in the Trigonometrical Survey, and will give the difference of longitude on a sphere.

But even the correct expression of the difference of longitude, which we have here investigated, has a disadvantage that makes it improper to be applied in practice; namely, the result is always affected with the sum of the errors of the two azimuths, which is likewise heightened by the divisor of the formula. In the instance of Beachy Head and Dumnose, we have

$$
\begin{array}{ll}
\lambda=50^{\circ} 44^{\prime} 21^{\prime \prime}, & m=96^{\circ} 55^{\prime} 58^{\prime \prime} \\
\lambda^{\prime}=50375 & m^{\prime}=815653:
\end{array}
$$

and with these data, the difference of longitude will be found equal to,

$$
1^{\circ} 26^{\prime} 54^{\prime \prime} \cdot 76 .
$$

Now I have computed the three following values of the same difference of longitude from independent data; one, by the formula (A) at p. 190 of the last Number of this Journal, which is independent of the azimuths; and two, by employing the formula $(B)$ at p. 191, combining the azimuth at one station with the latitude of the other, the result not being sensibly affected in this mode of computation by any probable error in the azimuth; viz.

$$
\begin{aligned}
& 1^{\circ} 27^{\prime} 5^{\prime \prime} \cdot 62 \\
& \begin{array}{lllll}
1 & 27 & 5 & \cdot 63
\end{array} \\
& \begin{array}{llll}
1 & 27 & 5 & \cdot 61
\end{array}
\end{aligned}
$$

It appears therefore that the result obtained by the formula investigated in this article is in defect about $11^{\prime \prime}$, which can only arise from an error of about $9^{\prime \prime}$ in the sum of the azimuths. J. Ivory.

XLIII. Some Remarks on an Article in the Bulletin des Sciences Mathematiques Physiques et Chimiques, for March 1828. By J. Ivory, Esq. A.M. F.R.S. \& .**

$\mathrm{N}$ the Bulletin des Sciences Mathematiques Physiques et Chimiques for March last, there is an article relating to the papers inserted in this Journal, which treat of the attraction of spheroids and the figure of equilibrium of a homogeneous planet in a fluid state. The remarks of the author on the opinions I have ventured to advance on these subjects, seem to call for some notice from me, which I shall study to make as brief as possible.

With regard to the attraction of spheroids, the usual ground of the dispute is shifted. The controversy has hitherto been confined to the law of attraction that prevails in nature; namely,

* Communicated by the Author.

when 